\title{
Chronic Myeloid Leukemia as Secondary Malignancy Following the Treatment of Hodgkin Lymphoma: A Case Series
}

\author{
RALPH MILLETT $^{1}$, ANITA AGGARWAL ${ }^{2}$, IMAD TABBARA ${ }^{1,3}$ and SAMAH NASSEREDDINE ${ }^{1,3}$ \\ ${ }^{1}$ The George Washington University School of Medicine, Washington, DC, U.S.A.; \\ ${ }^{2}$ The Department of Veteran Affairs Medical Center, Washington, DC, U.S.A.; \\ ${ }^{3}$ The George Washington Cancer Center, Washington, DC, U.S.A.
}

\begin{abstract}
Secondary malignancies are relatively common and clinically important phenomena following both chemotherapy and radiotherapy. The majority of these cases are acute leukemias, the occurrence of which have been thoroughly documented and studied. More rarely, chronic myeloid leukemias (CML) may arise subsequent to treatment of a primary malignancy. Literature review on such developments following treatment of Hodgkin's Lymphoma (HL) is scant. Herein, the authors present three cases of CML diagnosed within five years of treatment initiation for Hodgkin's Lymphoma (HL); one of the three patients had CML with atypical variant carrying a rare mutation with BCR-JAK2 fusion.
\end{abstract}

With an average five-year survival rate of $86.6 \%$, current firstline treatment regimens for Hodgkin's lymphoma (HL) have enabled significant advances in disease-free progression (1). As a result, sequelae of therapy have become both increasingly prevalent and relevant in the survivor population. The most common secondary malignancies following treatment of HL are acute myeloid leukemias (AML) and myelodysplastic syndromes (MDS) with an estimated prevalence of 0.8-6.3\% within 20 years of treatment (2). Development of chronic myeloid leukemia (CML) subsequent to treatment is a considerably rarer event, such that it remains infrequently reported in the literature. Complicating this, population and cytogenetic characteristics of primary and secondary CML appear largely homogenous, making it uncertain if reported cases represent true secondary malignancies or they are rather due to de novo mutations (3). Herein, we present three cases of CML detected after treatment for HL, two with $B C R-A B L$ mutations and a third case of a CML harboring a less common $J A K 2-B C R$ translocation.

Correspondence to: Samah Nassereddine, MD, Division of Hematology/Oncology at GW Cancer Center, 2150 Pennsylvania Avenue, NW, Washington, DC 20037, U.S.A. Tel: +1 2027412478, Fax: +1 2027412487, e-mail: Samahnd@gwu.edu

Key Words: CML, Hodgkin lymphoma, secondary malignancy.

\section{Case Series}

Patient 1, a 53-year-old man, was diagnosed with stage IIA nodular lymphocytic predominant HL with right axillary lymph node involvement. After completion of 6 cycles of Adriamycin/ Bleomycin/Vinblastine/Dacarbazine (ABVD) therapy a positron emission tomography (PET)/computed tomography (CT) demonstrated complete remission.

A total of 1.5 years after HL diagnosis, the patient developed an elevating white blood cell (WBC) count from $15.3 \mathrm{k}$ to $118 \mathrm{k}$ over four months. Fluorescence in situ hybridization (FISH) on peripheral blood was positive for a $B C R-A B L 1$ e $13 \mathrm{a} 2$ fusion transcript $(60.14 \%)$ indicating the development of CML. He was started on hydroxyurea and allopurinol before being transitioned to imatinib. He reached complete hematological and cytogenetic response though only attained partial molecular response.

Patient 2, a 62-year-old man with a family history of paternal gastric cancer, fraternal granulocytic leukemia, and sororal breast cancer, was diagnosed with stage IIA Classical HL nodular sclerosis type after noticing a left-sided neck mass. The patient responded well to 6 cycles of ABVD therapy. Repeat PET/CT imaging one year later was without evidence of recurrent disease. The patient remained symptomfree for approximately two years after completion of therapy.

Three years after initial HL diagnosis, routine blood work demonstrated uptrending WBC (24.8k) and platelet (558k) counts. PET/CT revealed diffuse hypermetabolic bone marrow activity concerning for a primary bone marrow disorder. FISH revealed a BCR-ABL1 e14a2 fusion transcript $(90.31 \%)$ as well as a BCR-ABL1 e13a2 fusion transcript $(2.42 \%)$ and the patient was diagnosed with CML. He was started on imatinib and reached major molecular response.

Patient 3 is a 53-year-old undomiciled man who was diagnosed with HL. He received two cycles of ABVD and was lost to follow-up shortly thereafter. He presented to clinic several months later at which time PET/CT imaging demonstrated a hypermetabolic left axillary lymph node (maximum SUV 19.8) and bilateral inguinal lymphadenopathy. A biopsy of the axillary lymph node revealed nodular 
lymphocyte predominant HL (NLPHL). A mild obstructive defect on pulmonary function tests, a decreased DLCO, and a multi-gated acquisition study with an estimated ejection fraction of $49 \%$ precluded further ABVD therapy. Instead, the patient was started on Rituximab/Etoposide/ Prednisolone/ Oncovin/Cyclophosphamide/Hydroxydaunorubicin (REPOCH) therapy. He received a total of three cycles before the regimen became intolerable, followed by radiation therapy to the residual disease in the left axilla.

Four years after, the patient returned to clinic with progressive anemia. A bone marrow biopsy demonstrated hypercellular marrow with trilineage hematopoiesis, left shift myelopoiesis, and diffuse grade II-III myelofibrosis. Subsequent karyotype was negative for translocation $(9,11)$ and FISH studies were negative for BCR-ABL. A JAK2BCR fusion was suspected based on PCR and FISH analysis revealing a translocation $\mathrm{t}(9 ; 22)(\mathrm{p} 24 ; \mathrm{q} 11.2)$. A biopsy of a left inguinal lymph node was without evidence of HL but did show follicular hyperplasia and progressive transformation of germinal centers. The patient was diagnosed with CML with atypical variant and began imatinib and erythropoietin therapy without any improvement. He is currently being evaluated for stem cell transplant.

\section{Discussion}

The development of secondary CML has been reported in the setting of HL, NHL, and other lymphoid malignancies but it remains an uncommon event. Further, given the cytogenic and demographic similarity between de novo CML and secondary CML (3), it is difficult to discern if the abovereported cases are actually related to treatment of HL. In determining a relationship, several considerations must be made. These include the primary malignancy treated, the time between treatment inception and detection of CML, and possible mechanisms for the development of a secondary malignancy.

Regarding primary malignancy, all of the above cases of CML followed treatment of HL. One comprehensive review of secondary CML demonstrates that it is, in fact, more commonly attributed to treatment of HL than any other single malignancy $(>25 \%)$. Of note, however, no individual study in this set demonstrated a statistically significant excess of CML compared to control populations (3). No mechanistic explanation for this was proffered, though it was suggested that this represents both a reporting bias (the seeming uncommonness of this event leading to overreporting) as well as a detection bias (patients already being followed by a hematologist/oncologist are more likely to have other malignancies detected).

That said, there does appear to be a fairly consistent temporal relationship between treatment of $\mathrm{HL}$ and the development of CML. In the aforementioned review, that average difference was approximately four years. In our three cases, CML was detected within three years of initial treatment for HL. While it remains difficult to determine the precise chance of developing two unrelated primary malignancies within such a short time, the apparent consistency and short interval do suggest a relationship between treatment inception and development of CML.

As for the possible mechanism of secondary CML development, there does not appear to be an obvious increase in cases of CML after treatment with chemotherapy alone. Compared to AML and MDS for which exposure to DNAdamaging medications comprise between 10-20\% of cases, rates of all secondary CML appear to increase only in regimens involving radiotherapy (4). Previous studies have demonstrated a $0.7 \%$ chance of developing CML after ABVD therapy alone as opposed to a $9.5 \%$ chance after combined ABVD and radiotherapy $(5,6)$. This may be attributed to the relatively increased amount of time that the progenitor stem cells of CML spend in a quiescent phase, especially compared to the fast-replicating progenitor cells of AML and MDS $(3,4)$. Whereas intercalating regimens are most effective at addressing actively dividing cells, radiation has been shown to produce double-strand breaks even in cells in the $G_{0}$ and $G_{1}$ phases (4). Interestingly, only one patient in our cases received ionizing radiation therapy though all did receive at least partial ABVD treatment.

Given the limited number of cases presented here, as well as the relative scarcity of reported secondary CML in the literature, it is not possible to determine whether the above represent true secondary malignancies. It is, however, likely that the prevalence of such disease is higher than previously reported due to underdetection. Given the increasing rates and duration of disease-free survival with first-line HL treatment, this case series serves to highlight the necessity of close surveillance of post-treatment HL patients for the development of treatment-related malignancies.

Interestingly, the third case represents by itself a very rare form of CML with atypical variant. While the hallmark of CML is a $B C R-A B L$ translocation, in this instance the patient possessed a $B C R-J A K 2$ translocation $\mathrm{t}(9 ; 22)$ (p24;q11.2). Literature on such transformations remains sparse, however this specific translocation has been previously associated with both CML- and AML-like diseases (7-9). Novel fusions are being increasingly implicated in leukemias, including other $J A K 2$ variants such as ETV6-JAK2 fusions. BCR-JAK2 fusions, however, appear to be rare events, possibly due to lack of routine testing for these mutations. In one reported case a BCRJAK2 disease was not responsive to imatinib, likely due to lack of an active binding site for imatinib on an ABL kinase region (10). This serves to demonstrate that this fusion product may drive leukemic disease on its own and present a possible target for future therapies. 


\section{Conflicts of Interest}

The Authors declare that they have no financial relationship with any entity and that this manuscript is a reflection of their own work.

\section{Authors' Contributions}

All Authors contributed to the design, implementation, writing and reviewing of the manuscript.

\section{References}

1 Noone A, Howlader N, Krapcho M, Miller D, Bishop K, Kosary CL, Yu M, Ruhl J, Tatalovich Z, Mariotto A, Lewis DR, Chen HS, Feuer EJ and Cronin KA (eds.): SEER Cancer Statistics Review 1975-2014. SEER, National Cancer Institute, 2018.

2 Bhatia S: Therapy-related myelodysplasia and acute myeloid leukemia. Semin Oncol 40(6): 10, 2013. PMID: 24331189. DOI: 10.1053/j.seminoncol.2013.09.013

3 Aguiar RC: Therapy-related chronic myeloid leukemia: An epidemiological, clinical and pathogenetic appraisal. Leuk Lymphoma 29(1-2): 17-26, 1998. PMID: 9638972. DOI: 10.31 09/10428199809058378

4 Gale R, Hlatky L, Sachs R and Radivoyevitch T: Why is there so much therapy-related AML and MDS and so little therapyrelated CML? Leuk Res 38(10): 1162-1164, 2014. PMID: 25175829. DOI: 10.1016/j.leukres.2014.08.002

5 Valagussa P, Santoro A, Fossati-Bellani F, Banfi A and Bonadonna G: Second acute leukemia and other malignancies following treatment for Hodgkin's disease. J Clin Oncol 4(6): 830-837, 1986. PMID: 3711960. DOI: 10.1200/JCO. 1986.4. 6.830
6 Valagussa P, Santoro A, Fossati-Bellani F, Franchi F, Banfi A and Bonadonna G: Absence of treatment-induced second neoplasms after ABVD in Hodgkin's Disease. Blood 59(3): 488-494, 1982. PMID: 6174160.

7 Cuesta-Domínguez Á, Ortega M, Ormazábal C, Santos-Roncero M, Galán-Díez M, Steegmann JL, Figuera Á, Arranz E, Vizmanos JL, Bueren JA, Río $P$ and Fernández-Ruiz E: Transforming and tumorigenic activity of JAK2 by fusion to BCR: Molecular mechanisms of action of a novel BCR-JAK2 tyrosine-kinase. PLoS One 7(2): e32451, 2012. PMID: 223842 56. DOI: 10.1371/journal.pone.0032451

8 Elnaggar M, Agersborg S, Sahoo T, Girgin A, Ma W, Rakkhit R, Zorrilla I and Leal A: BCR-JAK2 fusion as a result of a translocation in a patient with CML-like myeloproliferative disease. Mol Cytogenet 5(1): 23, 2012. PMID: 22549126. DOI: 10.1186/1755-8166-5-23

9 He R, Greipp P, Rangan A, Mai M, Chen D, Reichard KK, Nelsen LL, Pardanani A, Hanson CA and Viswanatha DS: BCRJAK2 fusion in a myeloproliferative neoplasm with associated eosinophila. Cancer Genet 209(5): 223-228, 2016. PMID: 27134074. DOI: 10.1016/j.cancergen.2016.03.002

10 Griesinger F, Hennig H, Hillmer F, Podleschny M, Steffens R, Pies A, Wormann B, Haase D and Bohlande KS: A BCR-JAK2 fusion gene as the result of a $\mathrm{t}(9 ; 22)(\mathrm{p} 24 ; \mathrm{q} 11.2)$ translocation in a patient with a clinically typical chronic myeloid leukemia. Genes Chromosomes Cancer 44(3): 329-333, 2005. PMID: 160 01431. DOI: $10.1002 / \mathrm{gcc} .20235$

Received June 10, 2019

Revised June 24, 2019

Accepted June 27, 2019 